\title{
Neurotrophic Factor Expression After CNS Viral Injury Produces Enhanced Sensitivity to Psychostimulants: Potential Mechanism for Addiction Vulnerability
}

\author{
Marylou V. Solbrig,, ${ }^{1}$ George F. Koob, ${ }^{2}$ Loren H. Parsons,, ${ }^{2}$ Tomoko Kadota,, ${ }^{3}$ Nigel Horscroft, ${ }^{1}$ \\ Thomas Briese, ${ }^{1}$ and W. Ian Lipkin ${ }^{1}$ \\ 1 Departments of Neurology, Microbiology, and Molecular Genetics, University of California-Irvine, Irvine, California 92697- \\ 4292, ${ }^{2}$ Department of Neuropharmacology, The Scripps Research Institute, La Jolla, California 92037, and ${ }^{3}$ Department \\ of Anatomy, Chiba University School of Medicine, Chiba 260, Japan
}

Hypothesized risk factors for psychostimulant, amphetamine, and cocaine abuse include dopamine (DA) receptor polymorphisms, HIV infection, schizophrenia, drug-induced paranoias, and movement disorders; however, the molecular, cellular, and biochemical mechanisms that predispose to drug sensitivity or drive the development of addiction are incompletely understood. Using the Borna disease rat, an animal model of viralinduced encephalopathy wherein sensitivity to the locomotor and stereotypic behavioral effects of D-amphetamine and cocaine is enhanced (Solbrig et al., 1994, 1998), we identify a specific neurotrophin expression pattern triggered by striatal viral injury that increases tyrosine hydroxylase activity, an early step in DA synthesis, to produce a phenotype of enhanced amphetamine sensitivity. The reactive neurotrophin pattern provides a molecular framework for understanding how CNS viral injury, as well as other CNS adaptations producing similar growth factor activation profiles, may influence psychostimulant sensitivity.

Key words: virus; encephalitis; neurotrophin; Borna disease; rat; cocaine; amphetamine; dopamine
There is increasing experimental evidence to support a role for neuronal growth factors in the CNS response to drug exposure that outlasts the acute effects of drug. BDNF infusions into the ventral tegmental area (VTA) or nucleus accumbens (NAc) enhance cocaine-induced locomotor activity and conditioned reward in animal models (Horger et al., 1999). Factors neurotrophin-3 (NT-3) and basic FGF (bFGF) are increased in the VTA by repeated administration of psychostimulants (Flores et al., 1998; Pierce et al., 1999). Glial cell line-derived neurotrophin factor (GDNF) knock-out mice have greater sensitivity to locomotor and behavioral effects of cocaine (Messer et al., 2000). Growth factors, known to influence synaptic transmission (Boulanger and Poo, 1999), which act as sensitizing agents (Shu and Mendell, 1999) and regulate neuronal morphology (Connor and Dragunow, 1998), may mediate the increases in dendritic length, dendritic spine density, and numbers of branched spines on medium spiny neurons of the NAc that follow repeated D-amphetamine $(\mathrm{AMPH})$ or cocaine injections (Robinson and Kolb, 1997, 1999).

Borna disease virus (BDV) is a negative-strand RNA virus epidemiologically linked to patients with histories of intravenous drug abuse (Bode et al., 1988) and to dopaminergic syndromes such as schizophrenia (Hatalski et al., 1997). Adult rats infected

\footnotetext{
Received June 8, 2000; revised July 25, 2000; accepted Aug. 3, 2000.

This work was supported by National Institutes of Health Grant DA 00376 (M.V.S.) and NS 29425 (W.I.L.). We thank C. Ribak for helpful discussion and R. Kwon for technical assistance. Animal care and handling procedures were in compliance with institutional and National Institutes of Health guidelines. All experimental protocols and procedures were approved by the University of California-Irvine Institutional Animal Care and Use Committee. This is publication number 13021-NP from The Scripps Research Institute.

Correspondence should be addressed to Dr. Marylou Solbrig, Emerging Diseases Laboratory, Gillespie Neuroscience Research Building, Room 3107, University of California-Irvine, Irvine, CA 92697-4292. E-mail msolbrig@uci.edu.

Copyright (C) 2000 Society for Neuroscience $0270-6474 / 00 / 200001-05 \$ 15.00 / 0$
}

with BDV are sensitive to the locomotor and stereotypic behavioral effects of D-amphetamine and cocaine (Solbrig et al., 1994, 1998), have specific pathology in monoaminergic, prefrontal, and limbic circuits (Solbrig et al., 1994) and provide a unique experimental model for exploring mechanisms by which persistent CNS infection can modify psychostimulant sensitivity.

Drug sensitivity is one predictor of addiction vulnerability. Because of the BD rat's psychostimulant sensitivity, the CNS adaptations to virus are presumed similar to the adaptations to schedules of drug use that produce increases in amphetamine or cocaine sensitivity. Thus, the BD rat is used to elucidate links between cell physiology and reward circuit neuroadaptive mechanisms common to viral and repeated drug use insult.

Neurotrophins, which generate cascades of cytoplasmic signals, potentially influence cytoplasmic protein mediators of psychostimulant sensitivity through stimulated kinase reactions. One target for neurotrophin signaling may be tyrosine hydroxylase (TH) phosphorylation. TH is the rate-limiting step in dopamine (DA) synthesis; enzymatic activity is enhanced by multiple kinases acting at several phosphorylation sites. Increased TH activ-

This article is published in The Journal of Neuroscience, Rapid Communications Section, which publishes brief, peerreviewed papers online, not in print. Rapid Communications are posted online approximately one month earlier than they would appear if printed. They are listed in the Table of Contents of the next open issue of JNeurosci. Cite this article as: JNeurosci, 2000, 20:RC104 (1-6). The publication date is the date of posting online at www.jneurosci.org.

http://www.jneurosci.org/cgi/content/full/4627 
ity, in turn, would enhance dopaminergic pharmacological effects, such as those of the indirect DA agonists D-amphetamine and cocaine.

To test the hypothesis that neuronal growth factors contribute to the enhanced behavioral response to D-amphetamine and cocaine in BD rats, we examined neurotrophin transcript levels in striatum (caudate putamen and nucleus accumbens) and midbrain (substantia nigra pars compacta plus ventral tegmental area), neuropil microenvironments of DA termini and cell bodies, respectively. Using pharmacological and biochemical techniques to identify changes in DA synthetic steps, we suggest a specific neurotrophin expression pattern is related to increased $\mathrm{TH}$ function in surviving dopaminergic terminals.

\section{MATERIALS AND METHODS}

Subjects. Four-week-old male Lewis rats (Charles River Laboratories, Wilmington, MA) were intracerebrally infected with BDV by injection of $1.6 \times 10^{4}$ tissue culture infectious dose units, strain $\mathrm{He} / 80-1$, into the right lateral ventricle (BD rats) (Solbrig et al., 1994) or sham-infected with sterile PBS (NL rats). Six weeks later, infected rats and agematched, sham-infected rats were tested or killed. Animal care and handling procedures were in compliance with institutional and National Institutes of Health guidelines. All experimental protocols and procedures were approved by the University of California-Irvine Institutional Animal Care and Use Committee.

Tissue preparation. For nucleic acid, protein, and neurochemical analyses, rats were decapitated, and brains were removed. The striatum (caudate putamen and nucleus accumbens) was dissected from a 2-mmthick coronal section taken between 1 and $3 \mathrm{~mm}$ posterior to the anterior genu of the corpus callosum. A 1-mm-thick coronal section was taken through the mesencephalon at the superior colliculus, and a segment containing the substantia nigra pars compacta $(\mathrm{SNc})$ and VTA was dissected from the ventral portion of this block.

RNase protection assay analysis. RNA was isolated from striatal and mesencephalic regions homogenized in Tri-Reagent (Molecular Research Center, Cincinnati, OH). Quantitation of neurotrophic factor mRNAs was by multiprobe RNase Protection Assay (RPA) for rat mRNA (RiboQuant Multi-Probe RPA System; PharMingen, San Diego, $\mathrm{CA})$. Probes were synthesized and labeled by transcription in the presence of $\left[{ }^{32} \mathrm{P}\right] \mathrm{UTP}$. RPAs were performed on $10 \mu \mathrm{g}$ of total RNA using an RPAIII kit (Ambion, Austin TX). Protected fragments were resolved on a $5 \%$ denaturing polyacrylamide gel and analyzed using a Storm 840 PhosphorImager (Molecular Dynamics, Sunnyvale, CA). Host genes L32 and GAPDH transcript levels were used as controls ( $n=8$ per group).

Electron microscopy. Anesthetized rats were perfused with $0.1 \mathrm{M}$ phosphate-buffered $4 \%$ paraformaldehyde, $0.5 \%$ glutaraldehyde, $0.25 \%$ ethyl acetimidate, and $1 \mathrm{mM} \mathrm{CaCl}_{2}$. Brain tissue was removed, cut in 1 $\mathrm{mm}$ sections, post-fixed in $2 \%$ osmium tetroxide, block-stained in $4 \%$ uranyl acetate, dehydrated in graded ethanols, embedded, sectioned, and stained with uranyl acetate and lead citrate, and photographed at 25,000 or $50,000 \times$ (Kadota and Kadota, 1979) ( $n=6$ per group).

Drugs. Drugs used were $\alpha$-methyl-para-tyrosine $(\alpha \mathrm{m} \rho \mathrm{t})$ methyl ester $(5,10,20 \mathrm{mg} / \mathrm{kg}$, i.p.) (Sigma, St. Louis, MO) and D-amphetamine sulfate $(0.25,0.50,1.00 \mathrm{mg} / \mathrm{kg}$, s.c.) (Sigma) (Weissman et al., 1966; Cooper et al., 1972) dissolved in sterile saline.

Behavioral testing. A dose of $\alpha \mathrm{m} \rho \mathrm{t}(20 \mathrm{mg} / \mathrm{kg}$, i.p.) was established as the lowest dose to suppress locomotor response to $1.0 \mathrm{mg} / \mathrm{kg}$ D-amphetamine in four normal, uninfected rats. Thereafter, BD and additional normal uninfected rats received the threshold $(20 \mathrm{mg} / \mathrm{kg}$, i.p.) $\alpha \mathrm{m} \rho \mathrm{t}$ dose, followed by one of three subcutaneous doses $(0.25,0.50$, or $1.00 \mathrm{mg} / \mathrm{kg}$ ) of D-amphetamine or its vehicle control $4 \mathrm{hr}$ later. Animals were tested during $3 \mathrm{hr}$ testing sessions once per week. Behavior was monitored continually in $40 \times 25 \times 20 \mathrm{~cm}$ cages equipped with two equally spaced horizontal photocell beams across the long axis. Locomotor activity, quantified by numbers of cross-overs (the successive interruption of two photobeams) (Solbrig et al., 1994) was plotted against dose ( $n=7-8$ per group). Comparison $\mathrm{BD}$ and normal groups received only D-amphetamine.

TH protein determination. For Western blotting, isolated brain regions were homogenized in $(1: 4 \mathrm{w} / \mathrm{v}) 220 \mathrm{~mm}$ sodium acetate, $\mathrm{pH} 6.15$ (Hyland et al., 1996). Ten micrograms of protein samples were size-fractionated by $10 \%$ SDS-PAGE (Mini-Protean-3 cell system; Bio-Rad, Hercules,
CA). TH was quantified by Western blotting using rabbit anti-TH polyclonal antibody (AB151; Chemicon, Temecula, CA) at 1:5000 dilution, an HRP-conjugated secondary antibody with an ECL-Plus detection system (Amersham, Arlington Heights, IL), and phosphorimaging analysis (Storm 840 PhosphorImager, Molecular Dynamics). Actin detected by mouse anti-actin monoclonal antibody (MAB 1501; Chemicon) served as a control for protein concentration loaded ( $n=4-6$ per group).

For immunohistochemistry, anesthetized animals were perfused with buffered $4 \%$ paraformaldehyde; brains were removed, post-fixed, and cryoprotected. Twenty micromolar slide-mounted sections were immunostained with rabbit anti-TH serum (Protos Biotech, New York, NY) at 1:1000, a biotinylated anti-rabbit secondary antibody, enhanced with avidin-biotinylated enzyme complexes, and reacted in 3,3'diaminobenzidine (DAB) ( $n=3$ per group).

TH activity assay. TH activity was measured in soluble fractions of isolated brain regions by HPLC detection of L-DOPA. Striatal and mesencephalic sections were homogenized in $(1: 4 \mathrm{w} / \mathrm{v}) 10 \mathrm{~mm}$ potassium phosphate, $\mathrm{pH} 7.4$, then centrifuged at $14,000 \times \mathrm{g}$ in $10 \mathrm{~K}$ Nanosep tubes (Pall Filtron Corporation, Northborough, MA). Seven hundred microgram protein aliquots were incubated at $37^{\circ} \mathrm{C}$ for $10 \mathrm{~min}$ in $100 \mathrm{~mm}$ sodium acetate buffer, $\mathrm{pH}$ 6, in the presence of: L-tyrosine, D,L-6-methyl5,6,7,8-tetrahydropterine- $\mathrm{HCl}\left(6 \mathrm{MPH}_{4}\right)(\mathrm{Sigma})$, catalase (Boehringer Mannheim), an aromatic L-amino acid decarboxylase inhibitor: 3-hydroxybenzylhydrazine 2HCl (NSD-1015) (Research Biochemicals, Natick, MA), and ferrous ammonium sulfate (Naoi et al., 1988) $(n=6-7$ per group).

TH phosphorylation and immunoprecipitation. Neutralized acid extracts of striatal brain homogenates were subjected to direct-back or indirectback phosphorylation (Guitart and Nestler, 1989). Twenty microgram aliquots of neutralized supernatants were phosphorylated with $0.03 \mu \mathrm{g}$ of purified catalytic subunit of cAMP-dependent protein kinase (PKA-c) (Sigma) or $5 \mathrm{U}$ of PKA holoenzyme (Sigma) and $10 \mu \mathrm{M} \gamma^{32} \mathrm{P}-\mathrm{ATP}(10$ $\mathrm{Ci} / \mathrm{mmol}$; DuPont NEN, Boston, MA).

TH was immunoprecipitated with rabbit anti-TH polyclonal antibody (Chemicon) and Streptococcal Protein G (Sigma). ${ }^{32} \mathrm{P}$ incorporation by TH was quantified by phosphorimaging (Storm 840 PhosphorImager, Molecular Dynamics). Duplicate immunoprecipitate samples were run ( $n=4$ per group).

Statistical analysis. Group differences in neurotrophin mRNA, TH protein, and L-DOPA levels were analyzed by unpaired two-tailed $t$ tests, with significance set at $p<0.05$. Separate BD and NL rat populations were used for each experiment.

Behavioral data were analyzed for differences in response to D-amphetamine and the suppressant effects of $\alpha \mathrm{m} \rho \mathrm{t}$ in BD and normal rats. Two-way ANOVA was performed with (BD or normal) group as the independent variable and repeated measures on (D-amphetamine) dose. Three-way ANOVA was performed with (infected or noninfected) group and $(\alpha \mathrm{m} \rho \mathrm{t})$ treatment as the independent variables and repeated measures on (D-amphetamine) dose. Differences among groups or treatment conditions were verified by simple main effects, post hoc Newman-Keuls, or $t$ tests.

Differences in levels of TH back phosphorylation, as percentages of total phosphorylation per treatment group, were assessed by $\chi^{2}$ analysis.

\section{RESULTS}

Changes in neurotrophic factor expression accompany degenerative changes in the striatum of BD rats

Neurotrophin transcript levels

To test the hypothesis that neuronal growth factors along DA circuits contribute to the enhanced behavioral response to D-amphetamine in BD rats, we examined neurotrophin transcript levels in striatum and SNc. Striata of BD rats had significantly increased levels of mRNAs for all tested members of the nerve growth factor (NGF) family of growth factors (the neurotrophins): $\beta$ NGF, BDNF, NT-3, and NT-4; a significant increase in CNTF, a member of the neuropoietic cytokine family; and a significant decrease in GDNF mRNA levels, a member of the transforming growth factor family (Fig. $1 A)(n=8$ per group). The changes in transcripts encoding neuronal growth factors suggest widespread striatal plastic changes. BDNF, NT-3, NT-4, and $\mathrm{CNTF}$ all have trophic effects on DA-producing cells in vitro 

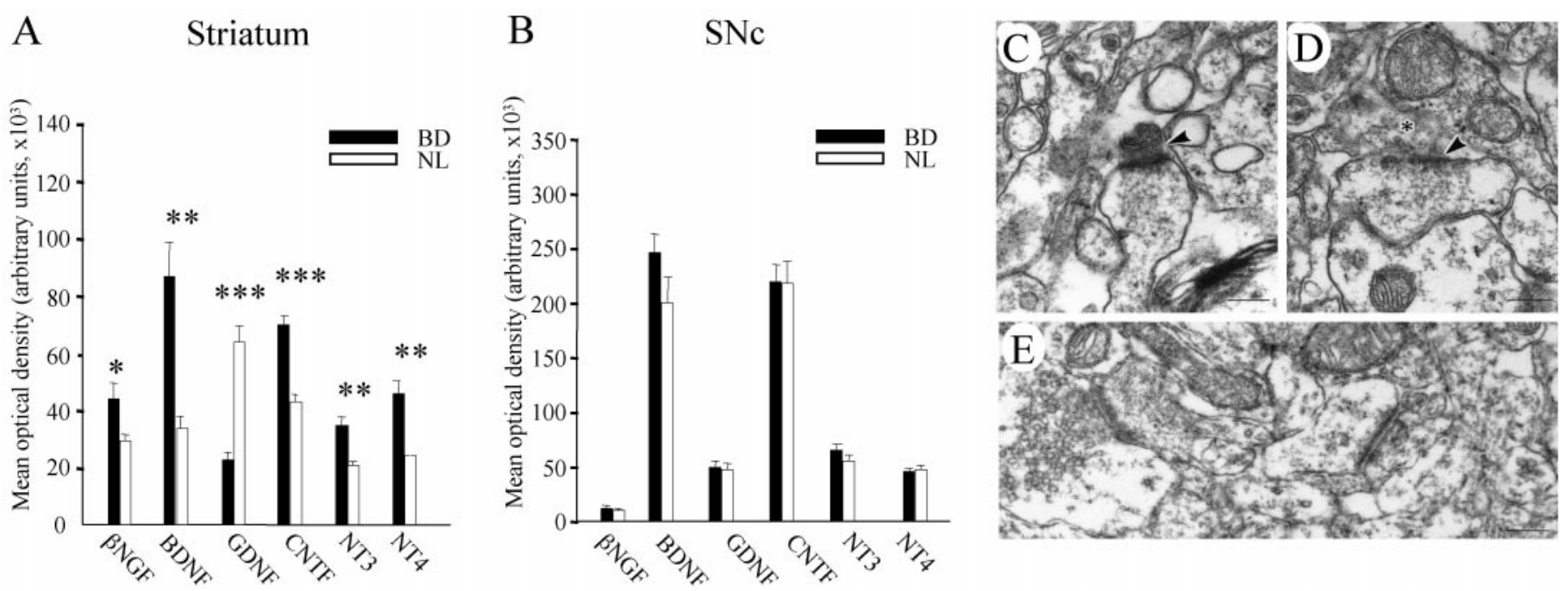

Figure 1. Regional neurotrophin expression and ultrastructure changes. $A, B$, Neurotrophic factor mRNAs were detected by multiprobe RPA (RiboQuant Multi-Probe RPA System; PharMingen) and quantified by PhosphorImager densitometry. There were significant increases in $\beta$ NGF, BDNF, CNTF, NT-3, and NT-4 mRNA levels and a significant decrease in GDNF mRNA in the striatum of BD rats $(A)$. SNc mRNA levels for neurotrophins were similar in BD and NL rats $(B)$. Values represent mean optical density in arbitrary units \pm SEM. Groups were compared by unpaired two-tailed $t$ tests with ${ }^{*} p<0.05,{ }^{* *} p=0.001$, and ${ }^{* * *} p<0.0001$, relative to normal uninfected rats $(n=8$ per group). $C-E$, Electron microscope study. Electron micrographs of the striatum of $\mathrm{BD}$ rats with uranyl acetate and lead citrate staining, illustrating degenerating axon terminals $(C, D$, arrows), and glial replacement of a presynaptic site $(D$, asterisk $)$. In $C$, the degenerating axon terminal is at the head of a dendritic spine. Normal rat striatum is pictured in $E$ (photographed at $50,000 \times$ ). Scale bar, $0.2 \mu \mathrm{m}$.

(Hagg and Varon, 1993; Connor and Dragunow, 1998). No group differences were found in neurotrophin mRNA content in SNc (Fig. 1B).

\section{Electron microscopy}

Ultrastructural changes in striatum were examined to evaluate integrity of axon terminals and synaptic sites related to DA (symmetric) synapses. Electron micrographs of the striatum in $\mathrm{BD}$ rats (Fig. $1 C, D$ ) showed degenerating axon terminals next to postsynaptic spines with thick postsynaptic densities. Figure $1 C$ illustrates a degenerating axon terminal at the head of a dendritic spine and a portion of a symmetric (DA) synapse on the neck of the same spine. Figure $1 D$ displays a vacated presynaptic site, now occupied by glia (asterisk), a finding consistent with axon terminal degeneration in this asymmetric (glutamatergic) synapse. For comparison, asymmetric synapses in the striatum of a normal uninfected rat appear in Figure $1 E$. Ultrastructural changes observed in the $\mathrm{BD}$ rat striatum are consistent with shifting distribution or survival of asymmetric (presumed excitatory, glutamatergic) and symmetric (presumed inhibitory, DA) synapses on dendritic spines of striatal neurons and are reminiscent of the plastic changes at points of convergence of dopaminergic and glutamatergic inputs hypothesized to contribute to the development of psychostimulant sensitization (White and Kalivas, 1998; Ghasemzadeh et al., 1999).

\section{Increased TH activity is also found in the striatum Behavioral pharmacology}

Behavioral supersensitivity to D-amphetamine is illustrated by a left shift in the D-amphetamine dose-response curve in BD rats (Fig. 2A). There was a significant difference in D-amphetamine locomotor activity by two-way ANOVA (group $\times$ AMPH dose, $\left.F_{(3,21)}=3.549 ; p<0.05\right)$ with significant increase in locomotor activity at $0.5 \mathrm{mg} / \mathrm{kg}$ dose for $\mathrm{BD}$ rats after BD dose main effect $\left(F_{(3,21)}=3.689 ; p<0.05\right)$.

Because growth factors have DA system maintenance and enhancing effects, we next tested whether the enhanced behavioral responses to D-amphetamine in BD rats (initially reported, Solbrig et al., 1994) is because of increased activity of the DA synthetic enzyme TH. Using pharmacological probes to block DA synthesis, we examined the locomotor response to AMPH in the presence of the TH inhibitor $\alpha \mathrm{m} \rho \mathrm{t}$. A $20 \mathrm{mg} / \mathrm{kg}$ (intraperitoneal) dose of $\alpha \mathrm{m} \rho \mathrm{t}$ was established as the lowest dose to suppress locomotor response to $1.0 \mathrm{mg} / \mathrm{kg}$ D-amphetamine in four normal, uninfected rats. Thereafter BD and additional normal uninfected rats received the threshold $(20 \mathrm{mg} / \mathrm{kg}$, i.p.) $\alpha \mathrm{m} \rho \mathrm{t}$ dose, followed by one of three subcutaneous doses $(0.25,0.50$, or $1.00 \mathrm{mg} / \mathrm{kg})$ of D-amphetamine or its vehicle control $4 \mathrm{hr}$ later. The suppressant effects of $\alpha \mathrm{m} \rho$ t were different in BD and normal rats. A significant difference in the effect produced by $\alpha \mathrm{m} \rho \mathrm{t}$ was revealed by threeway ANOVA (group $\times$ treatment $\times$ AMPH dose; $F_{(3,66)}=2.755$; $p<0.05)$. Although $\alpha \mathrm{m} \rho \mathrm{t}$ significantly attenuated AMPHinduced locomotion in NL rats $\left(1 \mathrm{mg} / \mathrm{kg} \mathrm{AMPH} ; F_{(1,11)}=5.034\right.$; $p<0.05$ ) (Fig. $2 B$ ), there was no effect of $\alpha \mathrm{m} \rho \mathrm{t}$ pretreatment in $\mathrm{BD}$ rats (Fig. $2 C$ ).

\section{TH protein and activity determination}

The possibility that differences in TH metabolism might underlie differences in the response to D-amphetamine was investigated by examining basal TH protein levels by immunostaining and Western blotting and HPLC measures of L-DOPA after timed reaction of L-tyrosine with striatal protein extracts. BD rat striatal sections had fewer stained $\mathrm{TH}$-immunoreactive fibers in the striatum, less intense staining of the fiber plexus, and greater prominence of myelinated tracts, consistent with dearborization and contraction of gray matter (data not shown). Levels of TH protein by Western blotting were significantly reduced in striatum of BD rats [BD $(0.3023 \pm 0.0324)$ vs NL $(0.5033 \pm 0.0583) ; t=3.009 ; \mathrm{df}=1.6$; $p<0.05]$. No group differences were found in TH content of SNc (Fig. 3A) ( $n=4-6$ per group).

$\mathrm{TH}$ activity was measured in soluble fractions of isolated brain 

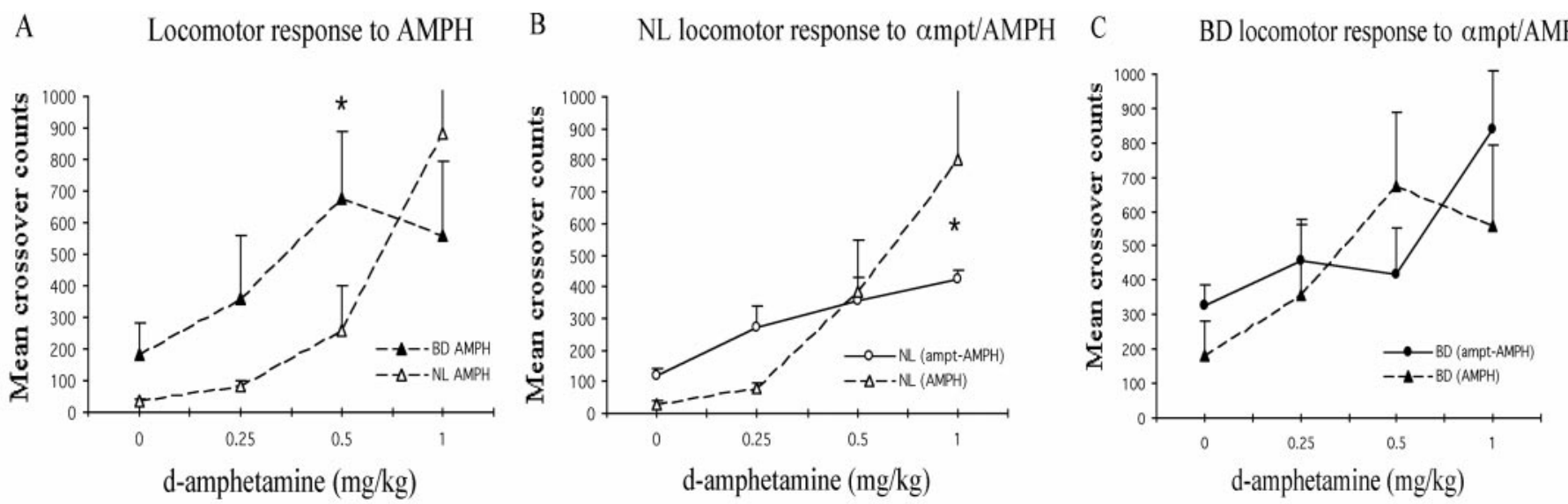

Figure 2. Effect of $\alpha \mathrm{m} \rho \mathrm{t}$ on AMPH-induced locomotor activity in noninfected (NL, panel B) and Borna-infected $(B D$, panel $C)$ rats. Animals were tested during $3 \mathrm{hr}$ testing sessions once per week. Locomotor activity, quantified in activity cages by numbers of cross-overs (the successive interruption of 2 photobeams) (Solbrig et al., 1994) was plotted against dose. D-amphetamine locomotor activity was significantly increased in BD rats. Individual means comparisons revealed a significant increase at $0.50 \mathrm{mg} / \mathrm{kg}$ dose $(A)$. In normal rats, D-amphetamine-induced locomotion was significantly decreased in the presence of $20 \mathrm{mg} / \mathrm{kg} \alpha \mathrm{m} \rho \mathrm{t}(B)$, whereas the same $\alpha \mathrm{m} \rho \mathrm{t}$ dose failed to suppress D-amphetamine locomotor activity in BD rats $(C)$. Values represent mean $( \pm \mathrm{SEM})$ locomotor counts over $180 \mathrm{~min} .{ }^{*} p<0.05$ ( $n=7-8$ per group).

A

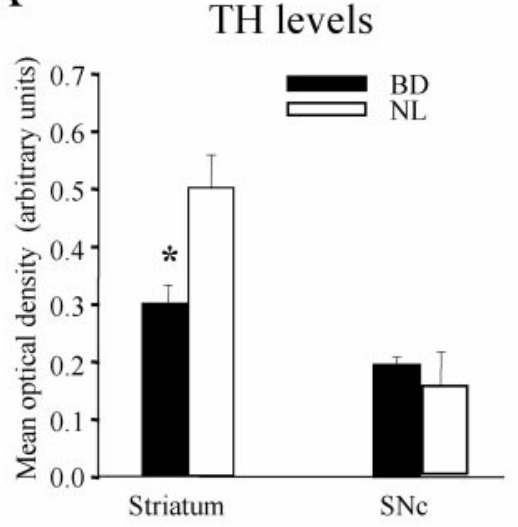

B

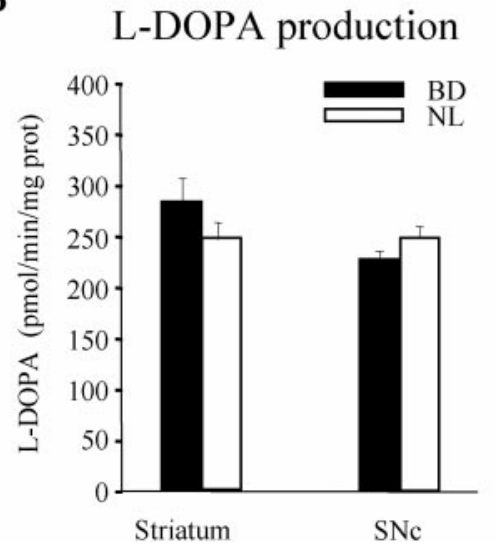

$\mathrm{C}$

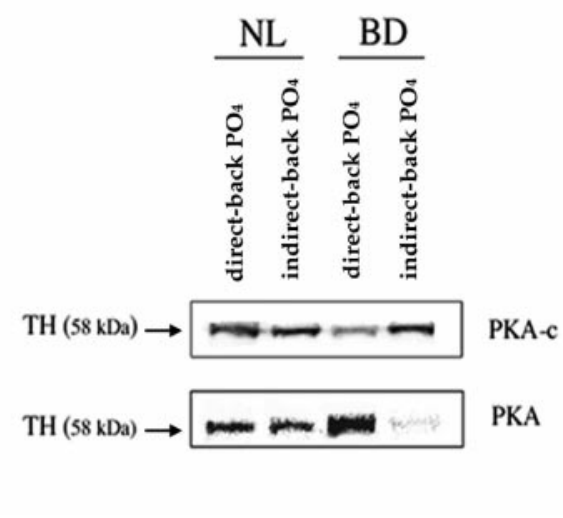

Figure 3. TH protein and activity studies. $A$, Regional changes in levels of TH protein, detected by Western blot using rabbit polyclonal antibody to $\mathrm{TH}$ (Chemicon AB151) and quantification by PhosphorImager densitometry. Levels of TH protein were significantly reduced in striatum of BD rats. No group differences were found in TH content of SNc. Values represent mean optical density in arbitrary units \pm SEM. ${ }^{*} p<0.05$ (two-tailed $t$ test) $(n=$ 4-6 per group). B, L-DOPA generated by incubations of striatal and SNc protein extracts with L-tyrosine, detected by HPLC. Despite significant reductions in striatal TH protein in $\mathrm{BD}$ rats $(A)$, no group differences were observed in the production of L-DOPA after 10 min incubations with 2 mM L-tyrosine and $700 \mu \mathrm{g}$ of striatal protein. Values represent L-DOPA produced (in picomoles per minute per milligram protein) \pm SEM. No group differences were found in L-DOPA production of SNc sections $(n=6-8$ per group). $C$, TH phosphorylation by PKA in vitro. Striatal protein extracts were incubated with $10 \mu \mathrm{M} \gamma^{32} \mathrm{P}-\mathrm{ATP}(10 \mathrm{Ci} / \mathrm{mmol}$; NEN) and $0.03 \mu \mathrm{g}$ of purified catalytic subunit of cAMP-dependent protein kinase (PKA-c) (Sigma), immunoprecipitated with anti-TH polyclonal antisera (Chemicon), size-fractionated by SDS-PAGE, and subjected to autoradiography and densitometric analysis. Levels of phospho-TH in striatum, produced by direct-back phosphorylation by the active catalytic subunit of PKA (PKA-c), were significantly lower in the BD rats $\left(66 \%\right.$; direct/indirect-back phosphorylation) versus $98 \%$ (direct/indirect-back phosphorylation) in NL group; $\chi^{2}=3.075 ; p<0.05$ (top panel). Results shown with these specimens are representative of results with other animals and consistent with increased basal phosphorylation at the Ser40 site in BD rats (see Results). A 1.75 times higher ${ }^{32} \mathrm{P}$ incorporation by $5 \mathrm{U}$ of PKA holoenzyme (Sigma) in BD rat direct-back phosphorylated specimens, indicated by immunoreactive bands (bottom panel), suggests the presence of elevated levels of cAMP to activate PKA.

regions by HPLC detection of L-DOPA. Despite significant reductions in striatal TH protein levels, L-DOPA levels generated by incubations of striatal protein extracts with L-tyrosine were equivalent in $\mathrm{BD}$ and normal rats after $10 \mathrm{~min}$ incubations with $0.2 \mathrm{~mm}$ L-tyrosine [BD $(98.29 \pm 8.11)$ vs NL (102.12 \pm 7.58); $\left.t_{(1,11)}=0.34 ; p=0.739\right]$ and increased, however, not significantly [BD $(284.45 \pm 24.02)$ vs NL $(248.57 \pm 16.06) ; p>0.05]$ in BD striatal preparations after incubations with $2 \mathrm{~mm}$ L-tyrosine (Fig. $3 B$ ). No group differences were found in L-DOPA production of SNc sections ( $n=6-8$ per group).

\section{Increased TH activity is related to increased phospho-TH}

\section{TH phosphorylation and immunoprecipitation}

TH phosphorylation at Ser40 correlates with increased TH activity in vivo; Phosphorylation at this site is dominated by cAMPdependent protein kinase A (PKA) (Haycock, 1990). TH phosphorylation status was examined, to determine the basis for retention of normal TH enzymatic activity despite reduced $\mathrm{TH}$ protein. Direct-back phosphorylation measures the dephospho form of a phosphoprotein; indirect-back phosphorylation provides an index of total protein by means of complete phosphor- 
ylation. Higher basal phosphorylation status results in a lower percentage of direct-back to indirect-back phosphorylation (Guitart and Nestler, 1989; Guitart et al., 1990). Levels of phospho$\mathrm{TH}$, produced by direct-back phosphorylation by the active catalytic subunit of PKA (PKA-c), were significantly lower in striatal brain homogenates of $\mathrm{BD}$ rats $(66 \%$; direct/indirect-back phosphorylation) versus $98 \%$ (direct/indirect-back phosphorylation) in NL group $\chi^{2}=3.075, p<0.05$ (Fig. 3C, top image), consistent with increased baseline levels of phospho-TH. Unexpectedly high ${ }^{32} \mathrm{P}$ incorporation was also found in the presence of (holoenzyme) PKA in the BD direct-back phosphorylation samples, suggesting the presence of elevated cAMP levels for the activation of PKA (Fig. 3C, bottom image). BD and normal uninfected samples had no intrinsic PKA activity, because no P incorporation was seen in protein extracts without PKA (data not shown). There were no significant differences in back phosphorylation in SNc samples treated with PKA-c or PKA (holoenzyme).

\section{DISCUSSION}

BDV infection of rats has pharmacological and lesion effects that result in behavioral sensitivity to psychostimulants. In this model of striatal injury, a specific neurotrophin expression pattern is associated with augmented DA synthetic activity.

One hypothesis to explain the mechanism of increased $\mathrm{TH}$ activity observed in the pharmacological and biochemical experiments is increased activity of neuronal growth factors of the NGF and neuropoietic cytokine families. Recently, BDNF and NGF were found to elevate cAMP and PKA in regenerating dorsal root ganglia cell cultures (Cai et al., 1999). Similarly, the capture and concentration of diff usable growth factors with cyclic nucleotide modulatory effects by presynaptic DA terminals could increase levels of activated PKA and increase basal TH activity derived from the TH Ser40 site. Our data are consistent with increases in basal TH phosphorylation at the Ser40 site, although phosphorylation at other sites by excess PKA has not been excluded. Evaluation of TH Ser31 site will be important because an NGF-sensitive phosphorylation site at TH Ser31 has been demonstrated on PC12 cells (Haycock et al., 1992). CNTF as well may have the ability to act or collaborate with other growth factors at the Ser31 site. The increase and prolongation of MAP kinase (ERK1 and 2) activity reported for differentiating sympathetic neurons in vitro by CNTF (Ip et al., 1994) may be relevant for the BD model, because ERKs phosphorylate TH at Ser31, a cellular response that would also enhance $\mathrm{TH}$ activity. Less predictable for enzymatic activity and expression of the syndrome is GDNF, whose transcripts are decreased in the striatum of BD rats. That GDNF uses two signaling pathways, the Ras/MAPK and phosphatidylinositol 3-kinase (PI3-kinase) pathways (Trupp et al., 1999), may prove important to understanding the biological and behavioral significance of its decrease in the BD rat.

We postulate that a specific neurotrophin expression pattern favors the development of drug sensitivity in the BD rat; the DA-enhancing effects of the neurotrophin and neuropoietic cytokine families are combined with the withdrawal of a protective role for GDNF. As drugs of abuse are recognized to have their own growth factor-enhancing effects, understanding the dynamics between neurotrophin and neurotransmitter systems and the optimum neurotrophin profile for clinical effects will provide insight into mechanisms of drug sensitivity, vulnerability, and addiction, and provide targets for therapeutic intervention. BDV has been linked to patients with psychiatric and drug abuse histories. The possibility that viral infection can mimic aspects of vulnerability to drug addiction raises the possibility that a history of viral challenge itself could result in enhanced vulnerability to drug addiction and speaks to models for future investigation of environmental insult, CNS adaptation, and drug addiction.

\section{REFERENCES}

Bode L, Riegel S, Ludwig H, Amsterdam JD, Lange W, Koprowski H (1988) Borna disease virus-specific antibodies in patients with HIV infection and with mental disorders. Lancet 2:689.

Boulanger L, Poo M (1999) Gating of BDNF-induced synaptic potentiation by cAMP. Science 284:1982-1984.

Cai D, Shen Y, DeBellard ME, Tang S, Filbin MT (1999) Prior exposure to neurotrophins blocks inhibition of axonal regeneration by MAG and myelin via a cAMP-dependent mechanism. Neuron 22:89-101.

Connor B, Dragunow M (1998) The role of neuronal growth factors in neurodegenerative disorders of the human brain. Brain Res Rev $27: 1-39$.

Cooper BR, Breese GR, Howard JL, Grant LD (1972) Enhanced behavioral depressant effects of reserpine and $\alpha$-methyltyrosine after 6-hydroxydopamine treatment. Psychopharmacologia (Berl) 27:99-110.

Flores C, Rodaros D, Stewart J (1998) Long-lasting induction of astrocytic basic fibroblast growth factor by repeated injections of amphetamine: blockade by concurrent treatment with a glutamate antagonist. J Neurosci 15:9547-9555.

Ghasemzadeh MB, Nelson LC, Lu X-Y, Kalivas PW (1999) Neuroadaptations in ionotropic and metabotropic glutamate receptor mRNA produced by cocaine treatment. J Neurochem 72:157-165.

Guitart X, Nestler EJ (1989) Identification of Morphine- and cyclic AMP-regulated phosphoproteins (MARPPs) in the locus coeruleus and other regions of rat brain: regulation by acute and chronic morphine. J Neurosci 9:4371-4387.

Guitart X, Hayward M, Nisenbaum LK, Beitner-Johnson DB, Haycock JW, Nestler EJ. (1990) Identification of MARPP-58, a morphine- and cyclic AMP-regulated phosphoprotein of $58 \mathrm{kDa}$, as tyrosine hydroxylase: evidence for regulation of its expression by chronic morphine in the rat locus coeruleus. J Neurosci 10:2649-2659.

Hagg T, Varon S (1993) Cliiary neurotrophic factor prevents degeneration of adult rat substantia nigra dopaminergic neurons in vivo. Proc Natl Acad Sci USA 90:6315-6319.

Hatalski CG, Lewis AJ, Lipkin WI (1997) Borna Disease. Emerg Infect Dis 3:129-135.

Haycock JW (1990) Phosphorylation of tyrosine hydroxylase in situ at serine 8, 19, 31 and 40. J Biol Chem 265:11682-11691.

Haycock JW, Ahn NG, Cobb MH, Krebs EG (1992) ERK1 and ERK2, two microtubule-associated protein 2 kinases, mediate the phosphorylation of tyrosine hydroxylase at serine-31 in situ. Proc Natl Acad Sci USA 89:2365-2369.

Horger BA, Iyasere CA, Berhow MT, Messer CJ, Nestler EJ, Taylor JR (1999) Enhancement of locomotor activity and conditioned reward to cocaine by brain-derived neurotrophic factor. J Neurosci 19:4110-4122.

Hyland K, Genasekera RS, Engle T, Arnold LA. (1996) Tetrahydrobiopterin and biogenic amine metabolism in the hph-1 mouse. J Neurochem 67:752-759.

Ip NY, Boulton TG, Li Y, Verdi JM, Birren SJ, Anderson DJ, Yancopoulos GD (1994) CNTF, FGF, and NGF collaborate to drive the terminal differentiation of MAH cells into postmitotic neurons. Neuron 13:443-455.

Kadota T, Kadota K (1979) Filamentous contacts containing subjunctional dense lattice and tubular smooth endoplasmic reticulum in cat lateral geniculate nuclei. Brain Res 177:49-59.

Messer CJ, Eisch AJ, Carlezon Jr WA, Whisler K, Shen L, Wolf DH, Westphal H, Collins F, Russell DS, Nestler EJ (2000) Role for GDNF in biochemical and behavioral adaptations to drugs of abuse. Neuron $26: 247-257$

Naoi M, Takahashi T, Nagatsu T (1988) Simple assay procedure for tyrosine hydroxylase activity by high-performance liquid chromatography employing coulometric detection with minimal sample preparation. J Chromatogr 427:229-238.

Pierce RC, Peirce-Bancroft AF, Prasad BM (1999) Neurotrophin-3 contributes to the initiation of behavioral sensitization to cocaine by activating the Ras/mitogen-activated protein kinase signal transduction cascade. J Neurosci 19:8685-8695.

Robinson TE, Kolb B (1997) Persistent structural modifications in nucleus accumbens and prefrontal cortex neurons produced by previous experience with amphetamine. J Neurosci 17:8491-8497.

Robinson TE, Kolb B (1999) Alterations in the morphology of dendrites and dendritic spines in the nucleus accumbens and prefrontal cortex following repeated treatment with amphetamine or cocaine. Eur J Neurosci 11:1598-1604.

Shu X-Q, Mendell LM (1999) Neurotrophins and hyperalgesia. Proc Natl Acad Sci USA 96:7693-7696.

Solbrig MV, Koob GF, Fallon JH, Lipkin WI (1994) Tardive dyskinetic 
syndrome in rats infected with Borna disease virus. Neurobiol Dis 1:111-119.

Solbrig MV, Koob GF, Lipkin WI (1998) Cocaine sensitivity in Borna disease virus infected rats. Pharmacol Biochem Behav 59:1047-1052.

Trupp M, Scott R, Whittemore SR, Ibanez CF (1999) Ret-dependent and -independent mechanisms of glial cell line-derived neurotrophic factor signaling in neuronal cells. J Biol Chem 274:20885-20894.
Weissman A, Koe BK, Tenen SS (1966) Antiamphetamine effects following inhibition of tyrosine hydroxylase. J Pharmacol Exp Ther 151:339-352.

White FJ, Kalivas PW (1998) Neuroadaptations involved in amphetamine and cocaine addiction. Drug Alcohol Depend 51:141-153. 